\title{
Antibiotic Resistant and Virulence Determinants of Staphylococcus haemolyticus C10A as Revealed by Whole Genome Sequencing
}

\author{
Kok-Gan Chan ${ }^{\circledR}$, Kim Tien Ng², Teik Min Chong¹, Yong Kek Pang², Adeeba Kamarulzaman², Wai-Fong \\ Yin ${ }^{1}$, Kok Keng Tee ${ }^{2}$ \\ 1. Division of Genetics and Molecular Biology, Institute of Biological Sciences, Faculty of Science, University of Malaya, Kuala Lumpur \\ 50603, Malaysia. \\ 2. Faculty of Medicine, University of Malaya, Kuala Lumpur 50603, Malaysia.
}

$\square$ Corresponding author: Division of Genetics and Molecular Biology, Institute of Biological Sciences, Faculty of Science, University of Malaya, Kuala Lumpur 50603, Malaysia; E-mail: kokgan@um.edu.my

(c) 2015 Ivyspring International Publisher. Reproduction is permitted for personal, noncommercial use, provided that the article is in whole, unmodified, and properly cited. See http://ivyspring.com/terms for terms and conditions.

Published: 2015.06 .03

\begin{abstract}
Staphylococcus haemolyticus is one of the pathogens that harbor a high level of antibiotic resistance. Here, we highlighted the potential determinants for multidrug resistance and virulence from the draft genome of Staphylococcus haemolyticus strain C10A, isolated from a patient with chronic obstructive pulmonary disease exacerbation.
\end{abstract}

Key words: Staphylococcus haemolyticus, whole genome sequencing, multidrug resistance, chronic obstructive pulmonary disease (COPD).

Chronic obstructive pulmonary disease (COPD), a chronic obstruction of lung airflow, is commonly associated with episodes of acute deterioration termed as "exacerbation" (1). Nearly $50 \%$ of COPD are due to viral infections, while the remaining are associated with bacterial infections $(2,3)$. Antibiotics are commonly prescribed for COPD exacerbations (4). As such, the emergence of multidrug resistant bacteria is possible, particularly in patients with recurrent COPD exacerbations.

Phenotypic assessment of resistance against commonly used antibiotics is widely practiced. However, such assessment is incapable of detecting the in vivo potential of the bacteria to escape the effect of antibiotics $(5,6)$. Recent advancement in next generation sequencing technologies has deemed to be a convenient and cost effective approach to assess the genomic properties of organisms (7). Here, based on genomic data generated from whole genome se- quencing (WGS), antimicrobial resistance profile of $S$. haemolyticus strain $\mathrm{C} 10 \mathrm{~A}$, isolated from the sputum of a 40-year-old male COPD subject with 20 years of smoking history was investigated in silico. During recruitment, the ratio of forced expiratory volume to forced viral capacity (FEV1/FVC) was less than $70 \%$ and the spirometry indicated a less than $12 \%$ of improvement of FEV1 after the bronchodilator test.

Isolation of the bacteria was performed using tryptic soy agar at $37^{\circ} \mathrm{C}$ followed by phenotypic identification using Microflex MALDI-TOF (Bruker Daltonik $\mathrm{GmbH}$, Leipzig, Germany) bench-top mass spectrometer. Classification of bacteria was then performed using the built-in Bruker MALDI Biotyper Real Time Classification software (Version 3.1, Build 65). Genomic DNA of strain C10A was extracted using MasterPure ${ }^{\mathrm{TM}}$ DNA Purification Kit (Epicentre Inc., Madison, WI, USA). Illumina MiSeq sequencing platform (Illumina, Inc., CA) was employed to se- 
quence the DNA library prepared using Nextera XT DNA sample preparation kit (Illumina, Inc., CA). The acquired sequence data was then subjected to quality trimming and de novo assembly with CLC Genomic Workbench (version 7.0). Sequence reads with low quality $(<\mathrm{Q} 20)$, ambiguous nucleotides and sequence length less than 50 nucleotides were discarded (8). For de novo assembly, contigs of at least $500 \mathrm{bp}$ and 30-fold coverage were selected. The assembled reads were subsequently subjected to annotation using NCBI prokaryotic annotation pipeline (PGAAP) in search of putative genes coding for antibiotic resistance.

With the spectra generated from mass spectrometry, the closest match of strain $\mathrm{C} 10 \mathrm{~A}$ was $S$. haemolyticus with the score value of 2.107 indicating secure genus and probable species identification. To gain further evidence of the bacterial identification, the closest neighbor displayed by RAST server (9) was S. haemolyticus JCSC1435, a multidrug resistant strain exhibiting high genomic plasticity mediated by numerous insertion sequences (10).

The draft genome of S. haemolyticus C10A was $2,430,312 \mathrm{bp}$ in size, with an average $\mathrm{G}+\mathrm{C}$ content of $32.6 \%$. Sequence assembly produced a total of 87 contigs with average size of $27,632 \mathrm{bp}$ and maximum contig size of $153,180 \mathrm{bp}$. The sequencing depth was at 74.1-fold. Results from PGAAP revealed a total of 2,387 coding sequences with 5 rRNAs and 57 tRNAs identified in the genome. The associated bioproject number for $S$. haemolyticus $\mathrm{C} 10 \mathrm{~A}$ genome is PRJNA246625.

In search of genes associated with resistance towards antimicrobial agents, the annotation revealed the presence of candidates against a wide spectrum of antibiotics including aminoglycosides, macrolides, quinolones, penicillins, tetracyclines and glycopeptides in the genome of S. haemolyticus strain C10A. Figure 1 demonstrated the clustering of these identified determinants together with a series of possible virulence factors including components of $c v f C$, heme oxygenases and peptidase U32, with other S. haemolyticus and Staphylococcus strains.

Genome sequencing and annotation of $S$. haemolyticus strain $\mathrm{C} 10 \mathrm{~A}$ in the present study has demonstrated the concise detection of multiple antibiotic resistance and virulence genes. Such observation has postulated the necessity to weigh the risk of antibiotic resistance, prior to its prescription for COPD patients.

Nucleotide sequence accession number: The genome sequence of S. haemolyticus $\mathrm{C} 10 \mathrm{~A}$ isolate has been deposited in GenBank under accession no.
JPRW01000000. The version described in this paper is the first version.

\section{Acknowledgement}

This research was financed by the University of Malaya High Impact Research (HIR) Grants (UM-MOHE HIR Grant UM.C/625/1/HIR/ MOHE/CHAN/14/1, no. H-50001-A000027; UM-MOHE HIR Grant UM.C/625/1/HIR/MOHE/ CHAN/01, No. A000001-50001) to Kok-Gan Chan and UM-MOHE HIR Grant UM.C/625/1/HIR/MOHE/ CHAN/2, no. H-50001-A000012 to Kok Keng Tee which are gratefully acknowledged.

\section{Ethic Statements}

The study was approved by the University Malaya Medical Centre (UMMC) Medical Ethics Committee. Standard, multilingual consent forms validated by the Medical Ethics Committee were used. Written consent was obtained from all study participants prior to collection of specimen.

\section{Competing Interests}

The authors have declared that no competing interest exists.

\section{References}

1. Mackay AJ, Hurst JR. COPD exacerbations: causes, prevention, and treatment. Med Clin North Am. 2012;96:789-809

2. Sethi S. Molecular diagnosis of respiratory tract infection in acute exacerbations of chronic obstructive pulmonary disease. Clin Infect Dis. 2011; 52 Suppl 4:S290-295

3. Sykes A, Mallia P, Johnston SL. Diagnosis of pathogens in exacerbations of chronic obstructive pulmonary disease. Proc Am Thorac Soc. 2007;4:642-646

4. Miravitlles M, Espinosa C, Fernandez-Laso E, Martos JA, Maldonado JA, Gallego M. Relationship between bacterial flora in sputum and functional impairment in patients with acute exacerbations of COPD. Study Group of Bacterial Infection in COPD. Chest. 1999;116:40-46

5. Garcha D, Donaldson G, McHugh T, Wedzicha J, Thurston S. Impact of antibiotic exposure on the development of antibiotic resistance in COPD patients. Microbiology. 2013;1: 2

6. Thurston SJ, Donaldson GC, McHugh TD, Wedzicha JA. Impact of COPD severity and sputum production on antibiotic resistance. Thorax. 2011;66(Suppl 4):A11-A11

7. Didelot X, Bowden R, Wilson DJ, Peto TE, Crook DW. Transforming clinical microbiology with bacterial genome sequencing. Nat Rev Genet. 2012;13:601-612

8. Chong TM, Yin WF, Mondy S, Grandclement C, Dessaux Y, Chan KG. Heavy-metal resistance of a France vineyard soil bacterium, Pseudomonas mendocina strain S5.2, revealed by whole-genome sequencing. J Bacteriol. 2012;194:6366

9. Aziz RK, Bartels D, Best AA, DeJongh M, Disz T, Edwards RA, et al. The RAST Server: rapid annotations using subsystems technology. BMC genomics. 2008:9:75.

10. Takeuchi F, Watanabe S, Baba T, Yuzawa H, Ito T, Morimoto $Y$, Kuroda M, Cui L, Takahashi M, Ankai A, Baba S, Fukui S, Lee JC, Hiramatsu K. Whole-genome sequencing of Staphylococcus haemolyticus uncovers the extreme plasticity of its genome and the evolution of human-colonizing staphylococcal species. J Bacteriol. 2005;187: 7292-7308. 


\section{Figures}

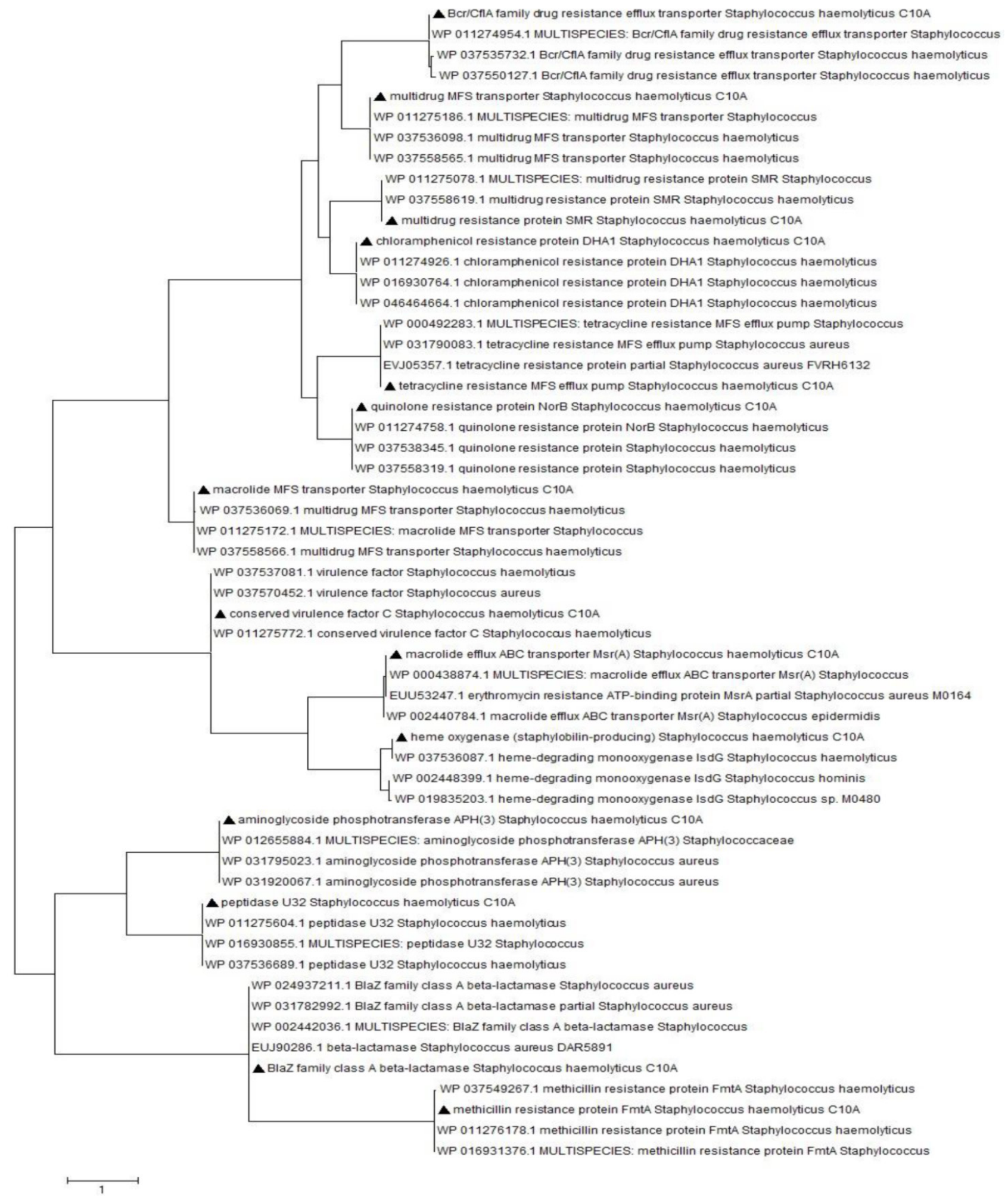

Figure 1: Phylogenetic analysis of putative genes associated with antibiotics resistance and virulence in the genome of S. haemolyticus strain C10A. Multiple sequence alignments was performed using ClustalW and evolutionary history was deduced using Maximum Likelihood method based on JTT matrix-based model. Triangles $(\boldsymbol{\Lambda})$ at each branches indicates the putative genes identified in the genome of S. haemolyticus strain C10A. 